\title{
Minimizing Delay and Maximizing Network Lifetime by Power-Aware Energy Efficient Routing [PAEER] Mechanism in Wireless Sensor Networks
}

\author{
Ramprakash ${ }^{\mathrm{a}, 1}$, Vijayakumari $\mathrm{B}^{\mathrm{b}}$ and Subathra $\mathrm{P}^{\mathrm{c}}$ \\ ${ }^{a}$ Research Scholar, CSE, University College of Engineering, Thirukkuvalai \\ ${ }^{b}$ Associate Professor, ECE, Mepco Schlenk Engineering College, Sivakasi \\ 'Professor, IT, Kamaraj College of Engineering and Technology, Viruthunagar
}

\begin{abstract}
We propose an efficient routing mechanism called PAEER(PowerAware Energy Efficient Routing) for meeting Network Lifetime Maximization and energy efficiency in the Wireless Sensor Networks(WSN).The different contributions of the PAEER approach are following (a) Multisink node approach which can lead to increase the nodes network lifetime and event detection mechanism that meets reliabilityrequirement of the WSN (b)Using PAEER mechanism sends the data to sink node by covering multi-path routes to aggregate the nodesdata. Thus energy consumption of the WSN canbe reducedmaximum level therefore network lifetime increased. This can be proved both theoretical and experiment solutions can be better when compared to other solutions. By using Network Simulator-3(NS-3) testbed the results show the better results for the all Quality of Service parameters(QoS) like Throughput, Network Lifetime, Power Consumption, etc.,
\end{abstract}

Keywords: Wireless Sensor Networks, Multisink nodes, Network Lifetime Maximization, PAEER

\section{Introduction}

A WSN is a collection of autonomous nodeswith limited constraints of energy capacities that must be a mobile node or stationary node and are located randomly in a dynamically changing environment. it is a low-cost, low energy consume network which can do the computation, storage, communication, and sensing, and acting through actuators. The energy consumption of a sensor node can be saved by intelligently controlling the mode of operation of each sensor node components. Here the energy efficiency is the energy required to transmit and receive a single bit of data. Sensor nodes operated on batteries have minimum capacity and recharging batteries for sensor nodes may be energy scavenging. Then the energy consumption of a sensor

\footnotetext{
${ }^{1}$ Ramprakash S, Research Scholar, CSE Department, University College of Engineering, Thirukkuvalai, E-mail: ramkeelai@gmail.com
} 
node must be tightly controlled. we described the related work, proposed work, testbed with conclusion are discussed.

\section{Related Work}

Energy is one of the most important resources wireless sensor networks. Therefore, the optimal use of energy is necessary.To improve energy-efficient nodes of WSNmay use node sleeping strategy to implement energy savings. Wireless sensor nodes run on batteries that are very difficult to recharge every time once it is deployed in the environment, this leadsto an energy efficiency problem in the network. Due to a battery constraint in wireless sensor networks presence for lifetime is essential. The protocol is reliable in terms of data reached at destination nodes. It's a collection of clustering protocol. A new energy-efficient clustering for WSN used to estimates the number of wakeup (active) nodes available in the network based on this hierarchy will find the optimality of clustering mechanism

The proposed PAEER mechanism compared with the existing protocols like Power Controlled Routing Protocol (PCRP) which is used to control the power in the adhoc networks.

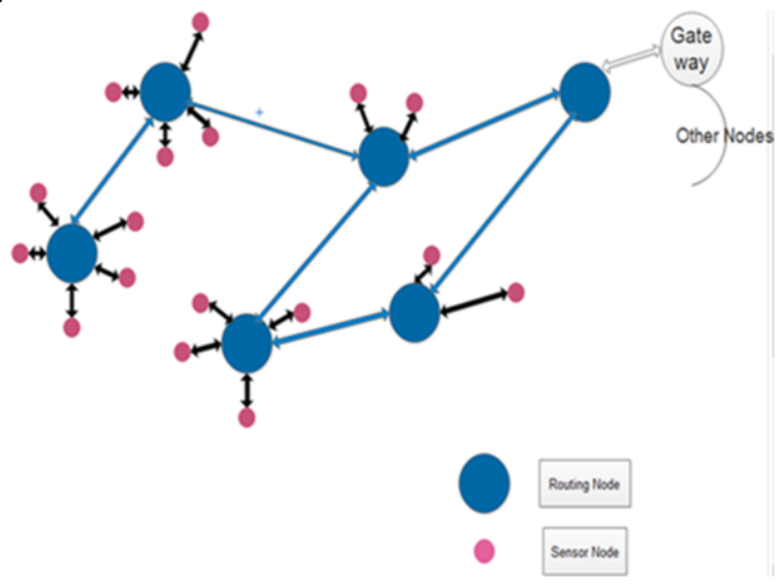

Figure 1. Routing mechanism in WSN

Grid Clustering Routing [GCR] which is used to create optimum clusters in terms of energyconsumption and PDORP Routingprotocol is to identify the dead nodes in the sensor networks.

To reduce the packet size of the network we go for a data aggregation technique. Many ways to implement aggregation.The first method isaggregation of data nodes that fuses the data gathered from different environmental sources that can be collectively organized and sends the final data in a compressed format which leads to reduce an error in data packets. Another method is to collective date from the above sources under single header forwards to the main cluster stationwithout affecting its originality of information.It also improves the accuracy of the data transfer. Many energy-efficient routing protocols can be proposed in wsn to minimizing total transmission overhead and improving the energy level of the wireless sensor networks. 


\section{Proposed Work}

\subsection{Multisink Node Mechanism}

Each WSN node partitions into clusters in which clusterhead and the other nodes of a clusters are member nodes. Thecluster head aggregates the data of its member nodes and transmits it to the sink node or to the other nodes for relaying in wireless sensor networks. Since the sink node is located at the longest distance from the cluster head, then the cluster head must spend significant energy for this transmission. If there are only some cluster heads the expected distance between a member node and its cluster head becomes longer and therefore the member node and its cluster head become longer and therefore the member has to spend more energy to reach its cluster head. Meanwhile multicluster spend more energy to reach its sink nodes.In our proposed system cluster head choose the energy-efficient routing path to choose optimal path to reach the desire sink nodes. here the routing setup path and route discovery taken place. By increasing the multi sink node the routing overhead can be reduced. Here the setup phase starts with the self-election of nodes to cluster heads. The advertisement phase starts the cluster heads advertise their neighborhood about information about the data transmission. So the multisink mechanism is the best way to determine the routing path in the sensor networks.

\subsection{PAEER Mechanism}

The lifetime of sensor nodes can be defined as the total time exhausted until the first sensor node in the network leakes its energy because once sensor nodes lost its energy it becomes in a dead condition. When its dead the sensing condition of the particular nodes in the network begins to degrade, therein network performances are ceased. Hereto maximize the network lifetime Power-Aware Energy Efficient Routing (PAEER) mechanism can be proposed. The proposed Energy efficient routing protocol lowering the number of Total transmission time between the nodes and forward the messages to all clustering nodes. The Total energy formula for transmission and reception messages is described as

$$
E_{T}=E_{\text {elec }} * \mathbf{m} * \mathbf{d}^{2}+E_{\text {elec }} * \mathbf{m}
$$

Here $E_{\text {elec }}$ is the energy consumption of the message $(m)$ with the distance $(d)$ between the sender and the receiver. By using the data aggregation mechanism we introduce a step-by-stepmultihop mechanism to select the k-multiple paths in the sensor networks. Each incoming data that is coming from the cluster head are aggregated then it is passed to the sink nodes here non-aggregate data are separated. The Energy consumption of the PAEER mechanism depends on three important factors.

1) Sensor nodes in the sleep mode

2) Sensor nodes in the active mode

3) Transmission of data between the sender and the receiver

\section{Performance Evaluation}

\subsection{Experimental Setup}

In this study, PAEER protocols are implemented from the default scratch file of Network Simulator version 3 (NS3). Applying various levels of QoS Parameters to the 
proposed system to evaluate performance of the Energy-efficient routing protocol by finding suitable simulator tool the most popular and advancement of next level simulator Network Simulator -3 (NS-3) is used.it is proposed to evaluate the performance in terms of time complexity, throughput, delay, packet delivery ratio and bit error rate etc...,

Table 1. Simulation entities

\begin{tabular}{|l|l|}
\hline & \\
Parameter & Values \\
\hline No of nodes for testbed & 200 \\
\hline Number of actual nodes taken for reference & 10 \\
\hline Retransmission range for the nodes & 5 \\
\hline Communication distance & $35 \mathrm{~m}$ \\
\hline Size of the Packet & $512 \mathrm{bytes}$ \\
\hline Bandwidth & $11 \mathrm{Mbps}$ \\
\hline Routing metric & ETX \\
\hline Elec Energy $\left(\mathrm{E}_{\text {elec }}\right.$ & $100 \mathrm{NJ} / \mathrm{bit}$ \\
\hline Amplifier energy $($ amp & \\
\hline Time interval $\left(\mathrm{I}_{\min }\right)$ & $200 \mathrm{PJ} / \mathrm{bit} / \mathrm{m}^{2}$ \\
\hline
\end{tabular}

\subsection{Performance Metrics}

1) Packet delivery ratio defines the total number of packets received by the sink nodes by the total number of packets sent

2) Energy consumption refers to the energy consumption of the nodes [Master and Slave].

3) Minimizing delay will improve the performance of a network

Delay nodes makeall possible timing delay during data transfer and reception of the nodes. This will depends on parameters like nodes waiting time, FIFO queuing order The testbed in the NS-3 Simulator shows the belowresults after simulating PowerAware Routing Algorithm (PAEER)in the scratch file. All simulations were conducted Ubuntu 18.04 operating system System with the necessary configurations, with the time slot of 3500 seconds of simulation time.

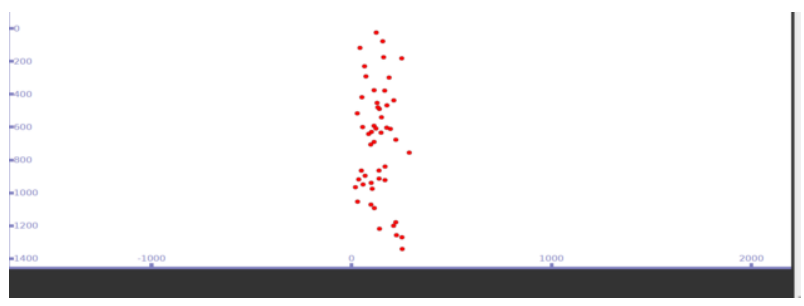

Figure 2. Initial Position of nodes 


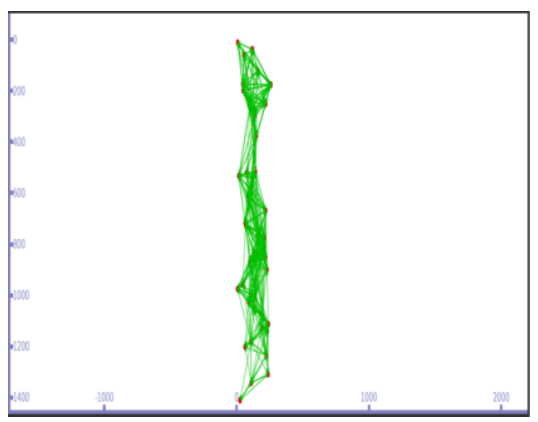

Figure 3. Connection establishment

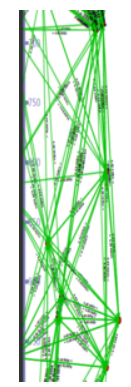

Figure 4. Data transmission

Comparing traditional routing protocols like GCR, PCRP, PDORP with the PAEER protocol. Comparison studies of the QoS parameters like throughput, power consumption with different values of sensor nodes.are depicted in the pictures below.Given the energy consumption parameter, PAEER will act as optimal energy routing protocol in the WSN. It has been observed from the following graphs.

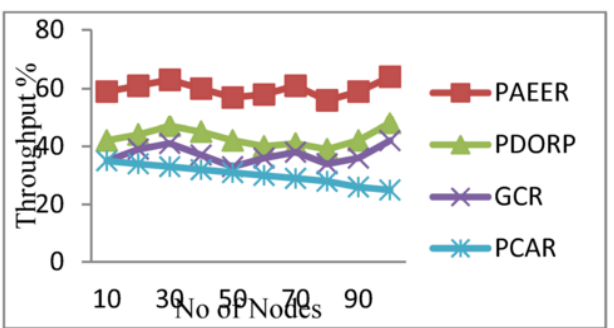

Figure 5. Throughput vs No of Nodes

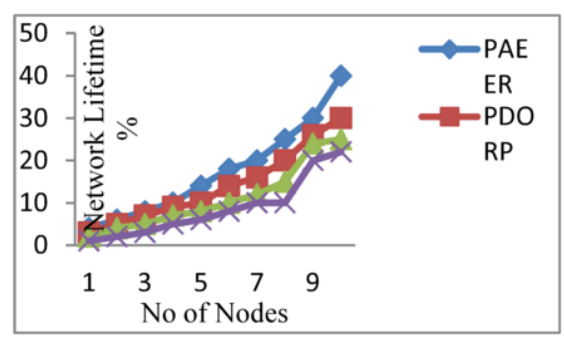

Figure 6. Network Lifetimevs Number of nodes

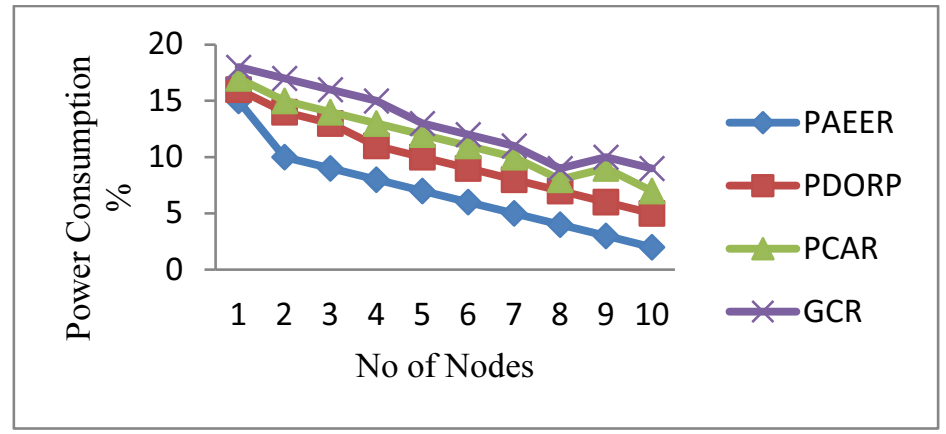

Figure 7. Power Consumption vs No of Nodes

\section{Conclusion}

We propose a novel routing scheme called PAEER (Power-Aware Energy Efficient Routing) for meeting better Energy Consumption and maximum lifetime as well as 
better reliabilityin the wireless sensor networks. Comparing with the traditional routing protocols, the PAEER mechanism ingeniously selects the cluster head far from the sink can select the optimal routing path mechanism and aggregates data before sending to the sink node. This approach can reduce both redundancy data transmission and the energy consumption of wsnnodes. Thus we can guarantee maximal lifetime and network reliability.

\section{References}

[1] Wireless sensor networks: A survey, Computer Networks,vol. 38, no. 4, pp. 393-422, March 2002.

[2] K. Romer and F. Mattern, .The design space of wireless sensor networks. IEEE Wireless Communications, vol. 11, no. 6, pp. 54-61,December 2004.

[3] D. Puccinelli and M. Haenggi, Wireless sensor networks: Applications and challenges of ubiquitous sensing.IEEE Circuits and Systems Magazine, vol. 5, no. 3, pp. 19-31, September 2005.

[4] V. Gungor and G. Hancke, Industrial wireless sensor networks:Challenges, design principles, and technical approaches. IEEE Transactions on Industrial Electronics, vol. 56, no. 10, pp. 42584265, October 2009.

[5] V. Gungor and G. Hancke, Industrial wireless sensor networks:Challenges, design principles, and technical approaches. IEEE Transactions on Industrial Electronics, vol. 56, no. 10, pp. 42584265, October 2009.

[6] T. Igoe, Making things talk: Practical methods for connecting physical objects. O'reilly, 2007.

[7] L. Atzori, A. Iera, and G. Morabito, .The internet of things: A survey.Computer Networks, vol. 54, no. 15, pp. 2787-2805, October 2010.

[8] Y. Chen and Q. Zhao, On the lifetime of wireless sensor networks.IEEE Communications Letters, vol. 9, no. 11, pp. 976-978, November 2005.

[9] R. Madan, S. Cui, S. Lall, and A. Goldsmith, Cross-layer design for lifetime maximization in interference-limited wireless sensor networks.IEEE Transactions on Wireless Communications, vol. 5, no. 11,pp. 3142-3152, November 2006.

[10]] J. W. Jung and M. Weitnauer, On using cooperative routing forlifetime optimization of multi-hop wireless sensor networks: Analysis and guidelines.IEEE Transactions on Communications, vol. 61, no. 8,pp. 3413-3423, August 2013.

[11]H. Yetgin, K. T. K. Cheung, M. El-Hajjar, and L. Hanzo, Cross-layer network lifetime maximization in interference-limited WSNs.IEEE Transactions on Vehicular Technology, vol. 64, no. 8, pp. 37953803, August 2015.

[12]] C. Cassandras, T. Wang, and S. Pourazarm, Optimal routing and energy allocation for lifetime maximization of wireless sensor networkswithnonideal batteries. IEEE Transactions on Control of Network Systems, vol. 1, no. 1, pp. 86-98, March 2014.

[13] Xue Zhang ; Ming Liu ; Haigang Gong," PCAR: A power controlled routing protocol for wireless ad hoc networks" IEEE access, 2010

[14] Seifemichael B. Amsalu, Wondimu K. Zegeyey, DerejeHailemariamz, YacobAstatkey,FarzadMoazzamiy," Energy Efficient Grid Clustering Hierarchy (GCH) Routing Protocol for Wireless Sensor Networks,IEEE Access,2016

[15] Gurbinder Singh Brar, Shalli Rani, Vinay Chopra, Rahul Malhotra,HoubingSong.Energy Efficient Direction-Based PDORP Routing Protocol for WSN, IEEE Special Section On Green Communications And Networking For 5G Wireless, 2016

[16]H. Yetgin, K. Cheung, M. El-Hajjar, and L. Hanzo,Network-lifetimemaximization of wireless sensor networks. IEEE Access, vol. 3, pp.2191-2226, November 2015.

[17] J. Du, K. Wang, H. Liu, and D. Guo, Maximizing the lifetime of k-discrete barrier coverage using mobile sensors. IEEE Sensors Journal,vol. 13, no. 12, pp. 4690-4701, December 2013.

[18] J. Chen, J. Li, and T. Lai, Trapping mobile targets in wireless sensor networks: An energy-efficient perspective. IEEE Transactions on Vehicular Technology, vol. 62, no. 7, pp. 3287-3300, September 2013

[19]J. Chen, J. Li, and T. Lai, Trapping mobile targets in wireless sensor networks: An energy-efficient perspective. IEEE Transactions on Vehicular Technology, vol. 62, no. 7, pp. 3287-3300, September 2013

[20]S. Soro and W. Heinzelman, Prolonging the lifetime of wireless sensor networks via unequal clustering.in IEEE International Parallel and Distributed Processing Symposium, Denver, CO, April 2005 . 
[21]D. Tian and N. D. Georganas, A coverage-preserving node scheduling scheme for large wireless sensor networks.in Proceedings of the 1st ACM international workshop on Wireless sensor networks and applications (WSNA), Atlanta, GA, USA, September 2002, pp. 32-41.

[22] W. Mo, D. Qiao, and Z. Wang, Mostly-sleeping wireless sensor networks: Connectivity, k-coverage, and -lifetime. in Proceedings of the 43rd Annual Allerton Conference on Communication, Control and

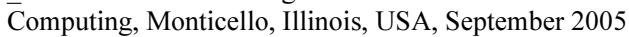

[23] M. Cardei, M. T. Thai, Y. Li, and W. Wu, Energy-efficient target coverage in wireless sensor networks.in 24th Annual Joint Conference of the IEEE Computer and Communications Societies (INFOCOM'05),vol. 3, Miami, FL, USA, March 2005, pp. 1976-1984.

[24] V.D.Ambeth Kumar (2017), Efficient Routing for Low Rate Wireless Network a Novel Approach.International Journal of Image Mining, Vol. 2, Nos. 3/4, 2017 